

\section{REVISTA \\ TEORÍA Y PRÁCTICA \\ DE LA \\ ARQUEOLOGÍA HISTÓRICA LATINOAMERICANA}

ISSN: 2250-866X (impreso) | ISSN: 2591-2801 (en línea)

AÑO VIII, VOLUMEN 9, PRIMAVERA DE 2019

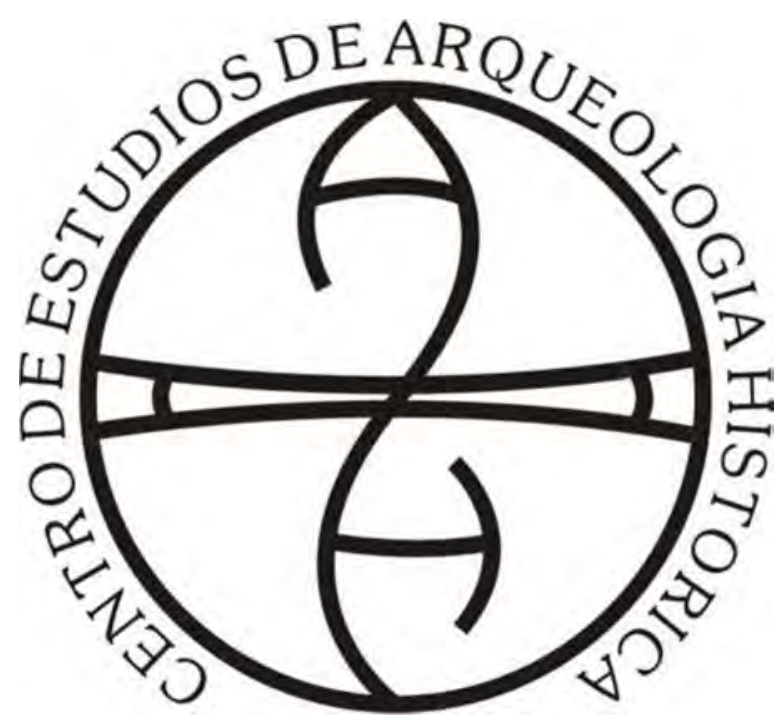

CENTRO de Estudios de ARqueOlOGía HistóricA

FACULTAD DE HUMANIDADES Y ARTES | UNIVERSIDAD NACIONAL DE ROSARIO 
(Universidad Nacional de Rosario, Universidad Nacional de Río Cuarto,

Universidad Nacional de San Juan, Universidad de la República, Universidad Nacional de Trujillo)

\author{
AUTORIDADES DE LA UNIVERSIDAD NACIONAL DE ROSARIO \\ RECTOR: Lic. Franco Bartolacci \\ VICE-RECTOR: Od. Darío Macía \\ SECRETARIO GENERAL: Prof. José Goity \\ SECRETARIA ACADÉMICO Y DE APRENDIZAJE: Dr. Marcelo Vedrovnik \\ SECRETARÍA DE CIENCIA TECNOLOGÍA E INNOVACIÓN \\ PARA EL DESARROLLO: Ing. Guillermo Montero.
}

\author{
AUTORIDADES DE LA FACULTAD DE HUMANIDADES Y ARTES \\ DECANO: Prof. Alejandro Vila \\ VICEDECANA: Prof. Marta Varela \\ SECRETARIA ACADÉMICA: Dra. Marcela Coria
}

\author{
AUTORIDADES DEL CENTRO DE ARQUEOLOGÍA HISTÓRICA \\ DIRECTORA: Dra. Ana Rocchietti \\ SECRETARIA: Prof. Nélida de Grandis \\ PROSECRETARIA: Lic. Marianela Bizcaldi
}

DIRECTORAS - EDITORAS:

Dra. Ana Rocchietti y Prof. Nélida De Grandis

SECRETARIA DE EDICIÓN: Dra. Irene Dosztal

Este número es co-edición de las ponencias

del VIII Congreso Nacional de Arqueología Histórica (2018) entre:

Centro de Estudios en Arqueología Histórica: Directora Ana Rocchietti

Centro de Estudios en Arqueología Regional: Director Fernando Oliva

Centro de Estudios en Arqueología Subacuática: Directora Mónica Valentini

Departamento de Arqueología, Escuela de Antropología: Director Fernando Oliva
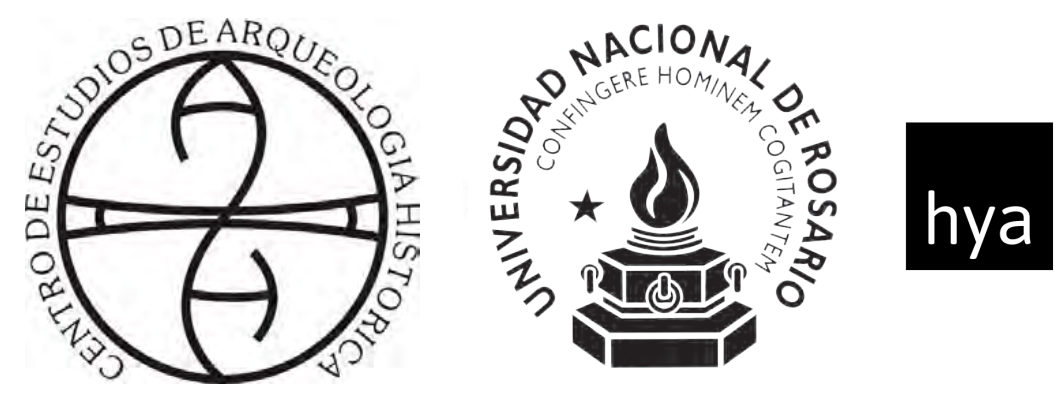

Facultad de Humanidades

y Artes_UNR
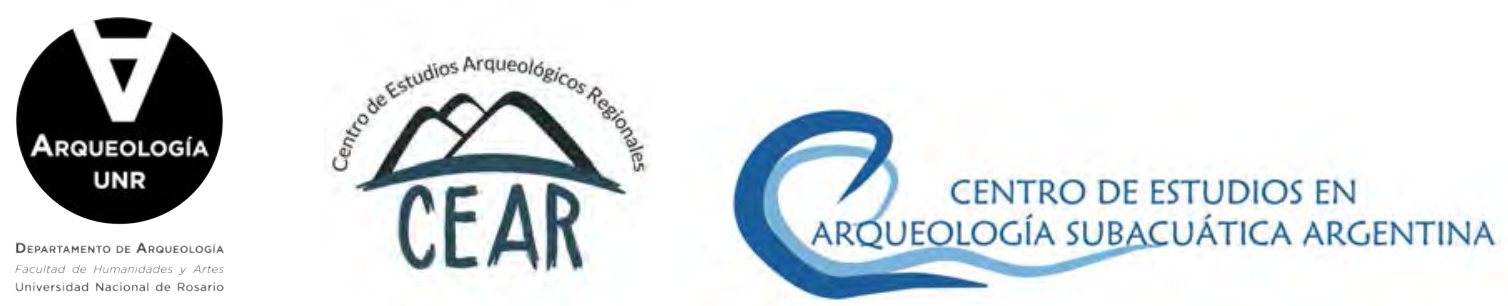
Comité Permanente de los Congresos Nacionales de Arqueología Histórica

Dr. Daniel Schávelzon (Universidad Nacional de

Buenos Aires)

Prof. María Teresa Carrara (Universidad Nacional de Rosario)

Prof. Carlos Baldassarre (Museo Municipal de Río

Grande, Tierra del Fuego) in memoriam

Dr. Mariano Ramos (Universidad Nacional de Luján, CONICET)

Dr. Horacio Chiavazza (Universidad Nacional de Cuyo)

Dra. Ana María Rocchietti (Universidad Nacional de

Rosario, Universidad Nacional de Río Cuarto)

Lic. Facundo Gómez Romero (Universidad Autónoma

de Barcelona)

\section{Comité Científico}

Dra. Tânia Andrade Lima (Universidade Federal do Rio de Janeiro)

Prof. Réginald Auger (CELAT/Département des

Sciences Historiques, Université Laval, Canadá)

Dr. Roberto Bárcena (Universidad Nacional de Cuyo, CONICET)

Dra. Marta Bonaudo (Universidad Nacional de Rosario, CONICET)

Dr. Leonel Cabrera (Universidad de la República, Uruguay)

Dr. Luis María Calvo (Universidad Católica de Santa

$\mathrm{Fe})$

Prof. Juan Castañeda Murga (Universidad Nacional de

Trujillo, Perú)

Dr. Carlos Ceruti (Museo de Ciencias Naturales y

Antropología "Prof. Antonio Serrano”. Paraná)

Dr. Horacio Chiavazza (Universidad Nacional de Cuyo)

Dra. Silvia Cornero (Universidad Nacional de Rosario)

Prof. Pedro Paulo Funari (Universidade Estadual de

Campinas, Brasil)

Lic. Jorge A. Gamboa Velásquez (Universidad Nacional

Santiago Antuñez de Mayolo, Perú)

Dr. Eduardo Alejandro García (Universidad Nacional de

San Juan, CONICET)

Prof. Nélida De Grandis (Universidad Nacional de

Rosario)

Dr. Juan Bautista Leoni (Universidad Nacional de

Rosario, CONICET)

Dra. Amancay Martínez (Universidad Nacional de San

Luis)

Dra. Catalina Teresa Michieli (Universidad Nacional de

San Juan, CONICET)

Lic. Fernando Oliva (Universidad Nacional de Rosario)
Ing. Adrián Pifferetti (Universidad Tecnológica Nacional Regional Rosario)

Dr. Mariano Ramos (Universidad Nacional de Luján, CONICET)

Dra. Ana María Rocchietti (Universidad Nacional de Rosario)

Dr. Daniel Schávelzon, (Universidad Nacional de Buenos Aires, CONICET)

Dra. Carlota Sempé (Universidad Nacional de La Plata)

Dr. Mario Silveira (Universidad Nacional de Buenos

Aires)

Dra. Silvia Simonassi (Universidad Nacional de Rosario)

Dra. Alicia Tapia (Universidad Nacional de Buenos Aires, Universidad Nacional de Luján)

Lic. Mónica P. Valentini (Universidad Nacional de Rosario)

Agrim. Benito Vicioso (Universidad Nacional de Rosario)

\section{Evaluaron este volumen}

Roberto Bárcena, María Teresa Boschin, Leonel Cabrera, Ulises Camino, María Rosa Carbonari, Carlos Ceruti, Horacio Chiavazza, Nicolás Ciarlo, Silvia Cornero, Eduardo Crivelli, Javier García Cano, Martín Gentinetta, María Laura Gili, Carlos Landa, Matilde Lanza, Melina Malandrino, Sebastián Pastor, Victoria Pedrotta, Josefina Piana, Mercedes Podestá, Mariano Ramos, Daniel Schavelzon, Diana Tamburini, Mónica Therrien, Mónica Valentini y María Teresa Boschin

\section{Diseño y diagramación}

Eugenia Reboiro

(eugenia.reboiro@gmail.com)

\section{Curadoría}

Ana Rocchietti e Irene Dosztal

Foto de tapa: Material arqueológico, del texto de Tapia et al.

\section{Propietario responsable:}

Facultad de Humanidades y Artes, Universidad Nacional de Rosario Centro de Estudios de Arqueología Histórica

Entre Ríos 758. Rosario, provincia de Santa

Fe (2000). Argentina.

Telf.: +54 (0341) 4802670

E-mail: ceahunr@gmail.com

Decreto Ley 6422/57 de Publicaciones

Periódicas 


\section{Índice}

Editorial

El origen del patrimonio histórico y su conservación integral en la contemporaneidad.

Yanina Aguilar

Prospección de basurales históricos de la ciudad de Rosario.

Soccorso Volpe y Gustavo Fernetti

Desconstrucción de un paisaje urbano. El Montevideo del extramuros colonial, aportes de la arqueología a su identidad actual. Ana Gamas

La Calota de Ameghino: reconsiderando un viejo tema desde la arqueología histórica.

Daniel Schávelzon

Análisis zooarqueológico de los restos hallados en una estructura sanitaria vinculada a momentos tempranos del actual barrio de Belgrano .71 Mario Silveira, Horacio Padula, Ricardo Orsini y Eva Bernat

La medida del tiempo: el reloj solar jesuita de La Cruz, provincia de Corrientes.

Fernando Oliva y María Cecilia Panizza

Sitio La Quinta: arqueología rural de campos serranos.

Flavio Ribero

Si no hay tabaco que no se note. Prácticas fumatorias en el fortin La Perra (1883-1885), La Pampa.

Alicia Tapia, Virginia Pineau y Melisa Ayelén Auge

El uso del tabaco y las pipas de caolin (clay pipes) en la frontera sur y oeste de la provincia de Buenos Aires en el siglo XIX.. .127

María del Carmen Langiano y Julio Fabián Merlo 
Patrimonio natural, urbano y arquitectónico de la costa en Mar del Plata. Federico Negroni

Huellas urbanas.

Ezequiel Serrot

Paisaje y patrimonio. La industria taninera en el siglo XX.

Provincia de Santa Fe.

Cristina Pasquali, Paola Milicic y Lara Ferré

Los desafíos de las arqueologías históricas latinoamericanas.

Andrés Zarankin

Sellos entre escombrales. Las lozas en el registro urbano

del Área Fundacional de Mendoza (siglo XIX y principios del siglo XX)

Lorena Puebla y Horacio Chiavazza

Evolución edilicia de la Plaza de Mayo de la Ciudad

de Buenos Aires (1580-1853).

Nicolás Ferrino

Gestión patrimonial en el rescate arqueológico y futuro Museo de Sitio

en Moreno 550, Ciudad Autónoma de Buenos Aires, Argentina.

María Eva Bernat, Ricardo Orsini, Horacio Padula y Mario Silvera 


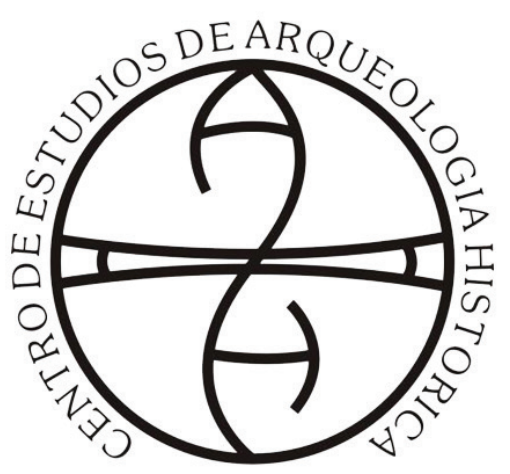

Centro de Estudios de Arqueología Histórica

Universidad Nacional de Rosario
Teoría y Práctica de la Arqueología Histórica

Latinoamericana | Año VIII. Vol. 9 | 2019

Revista del Centro de Estudios de Arqueología

Histórica, Facultad de Humanidades y Artes, Universidad Nacional de Rosario

https://teoriaypracticaah.unr.edu.ar/index.php/index ceahunr@gmail.com

ISSN en línea: 2591-2801

ISSN versión impresa: 2250-866X

\title{
Paisaje y patrimonio. LA INDUSTRIA TANINERA EN EL SIGLO XX. Provincia de Santa fe
}

\author{
Cristina Pasquali*, Paola Milicic** y Lara Ferré***
}

\section{Resumen}

Se presentan los modelos de Fichas de Inventario Patrimonial de las distintas secciones que conformaron el paisaje industrial desarrollado en el norte santafesino a fines del siglo XIX: Fábrica, Pueblo, Paraje, Estancia, Ferrocarril y Puerto. Los criterios de selección de los bienes inmuebles de cada sección se sustentaron en su asociación al paisaje industrial, al período histórico particular y al reconocimiento histórico y simbólico otorgado por los actores sociales de las comunidades implicadas.

Palabras clave: Industria, Patrimonio, Inventario

\section{Abstract}

The models of Heritage Inventory Sheets of the different sections that formed the industrial landscape developed in the north of Santa Fe at the end of the 19th century are presented: Factory, Town, Paraje, Cattle Ranch, Railway and Port. The criteria for the selection of real estate in each section were based on their association with the industrial landscape, the particular historical period and the historical and symbolic recognition granted by the social actors of the communities.

Keywords: Industry, Heritage, Inventory

\section{Introducción}

En este artículo se presentan los modelos de Fichas de Inventario Patrimonial de las distintas secciones que conformaron el paisaje industrial desarrollado en los departamentos de Vera y General Obligado (Provincia de Santa Fe) a fines del siglo XIX: Fábrica, Pueblo, Paraje, Estancia, Ferrocarril y Puerto

* CEAH - FhyA - UNR crispasquali@hotmail.com

** CEAH - FHyA - UNR paola.milicic@gmail.com

*** CEAH - FHyA - UNR laraferre88@gmail.com 
(Figura 1). ${ }^{1}$ La región actualmente es conocida como Cuña Boscosa y es el territorio más austral de la provincia fitogeográfica del Gran Chaco (Ragonese y Castiglioni, 1970). Desde mediados del siglo XIX profundas transformaciones políticas, sociales y económicas sentaron las bases para el establecimiento de redes ferroviarias, poblados ferroviarios, fábricas, “pueblos tanineros”, parajes, estancias y puertos con el objetivo de explotar los bosques de Quebracho colorado para la producción y exportación de extracto de tanino (Franchini y Roze, 1976). A partir de la década de 1950 comienza el cese de las actividades industriales tanineras en la provincia de Santa Fe con el cierre de la fábrica de Tartagal, finalizando dicho proceso en 1963, con la paralización de la fábrica de La Gallareta. Fallidos intentos de nuevas actividades industriales no subsistieron y provocaron la movilización de centenares de pobladores en búsqueda de nuevas alternativas laborales en las grandes ciudades. A pesar del tiempo transcurrido, ese pasado forma parte de las historias y problemáticas del presente.

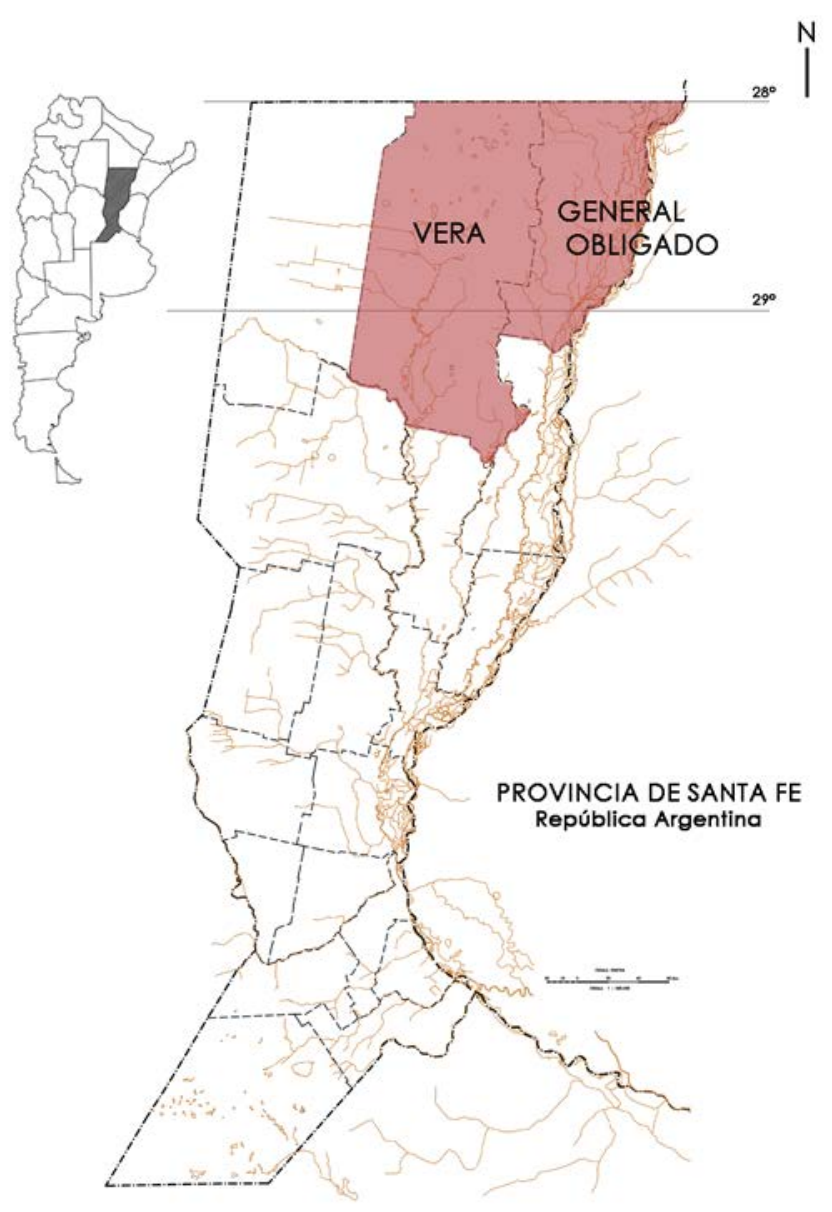

Figura 1. Área de investigación

\section{La conformación del paisaje industrial}

La conformación del paisaje industrial en el norte santafesino fue producto de varios factores que coadyuvaron para su génesis y se vincularon fundamentalmente a la propiedad de la tierra y a la explotación 
de los recursos naturales, especialmente, la madera del Quebracho colorado.

El proceso de configuración político, económico y social del territorio norte de la provincia de Santa Fe (actuales departamentos de Vera, General Obligado y 9 de Julio) se vincula, por un lado, a la formación del Territorio Nacional del Chaco (1872) y, por otro, a las consecuencias del empréstito Murrieta contraído por el gobierno provincial (1874).

Las incursiones militares iniciadas, a partir de 1870, posibilitaron el dominio del territorio al norte del arroyo del Rey (límite norte de la provincia de Santa Fe) y su organización política y administrativa. El control militar de las etnias chaqueñas y su incorporación como mano de obra facilitó la explotación de las riquezas naturales de la región. Numerosos obrajes se establecieron, a partir de la década de 1860, en puntos de fácil acceso fluvial sobre el río Paraná destinados al corte y labranza de maderas para durmientes, postes, subproductos como leña y carbón, como así también, para la exportación de rollizos de Quebracho colorado (Seelstrang, 1876; Carranza, 1884). ${ }^{2}$

El empréstito Murrieta tenía como destino la formación del Banco Provincial de Santa Fe y la construcción del Ferrocarril de Santa Fe a las Colonias del Oeste. Ante el incumplimiento del pago de las obligaciones, en 1881, el Gobierno provincial le "vende" a la compañía londinense, 479 leguas cuadradas de tierras provinciales y, un año después, otras 164 leguas de tierras públicas nacionales situadas al norte del paralelo $29^{\circ}$ de Latitud sur. La segunda venta se hizo bajo la condición que la provincia justificase el dominio sobre la tierra vendida, promoviéndose por tal motivo un expediente. En Agosto de 1883 se autoriza al Poder Ejecutivo nacional ratificar la venta hecha por el Gobierno provincial a Murrieta acordando previamente la forma en que debía hacerse el pago del precio hasta tanto se estableciera el límite norte. ${ }^{3}$ Finalmente, el Congreso Nacional aprueba, por medio de la Ley $N^{\circ} 1894 / 1886$, el tratado de límites entre las provincias de Santa Fe y Santiago del Estero. Se le otorga a Santa Fe el territorio nacional comprendido desde su límite norte (Arroyo del Rey) hasta el paralelo $28^{\circ}$ lo que significaba incorporar 500 leguas cuadradas y numerosas colonias: Ocampo (1878), Las Toscas (1882), Las Garzas (1883), Víctor Manuel (1883), Florencia (1884), San Antonio de Obligado (1884), Tacuarendí (1884), Piazza (1884), San Antonio de Obligado (1884) y Avellaneda (1886). Estos primeros proyectos de colonización e industrialización primaria tuvieron dos elementos fundamentales: sus propios puertos y ferrocarriles. Colonia Ocampo y Florencia construyeron sus respectivos sistemas logísticos mucho antes que se iniciara la industria taninera en la provincia de Santa Fe. Estas unidades de explotación económica constituyeron sus pequeños sistemas de transporte basados en puertos multipropósito y una pequeña red ferroviaria que los enlazaba con los enclaves residenciales, los solares productivos y las industrias asociadas (Maggi, 2014).

Paralelamente se estableció la red pública del Ferrocarril Santa Fe (F.C.S.F.) de Vera a La Sabana en el Territorio Nacional del Chaco (1892). Esta conexión norte-sur provincial permitió en un principio conectar obrajes y puertos para la exportación de rollizos y fue decisiva para estructurar y ordenar el territorio, como así también, para la posterior localización de los “pueblos tanineros”. Cabe destacar que la primera fábrica taninera establecida en la provincia de Santa Fe fue Calchaquí (1899) sobre la estación del F.C.S.F. del mismo nombre. ${ }^{4}$

Las fábricas/pueblos de Villa Guillermina (1904), La Gallareta (1906), Tartagal (c.1907) y Villa Ana (1910) fueron construidas por la Compañía Forestal del Chaco, Compañía Tanino de Santa Fe, Argentine Quebracho Company y The Forestal Land, Timber and Railways Company (en adelante The Forestal) respectivamente. Las compañías proyectaron y construyeron fábricas para la elaboración de extracto de tanino y pueblos en los contornos de los predios fabriles. Los pueblos contaban con viviendas y equipamientos colectivos para satisfacer las necesidades de abastecimiento, educación, salud y esparcimiento de la población, con el objetivo de atraer y fijar la mano de obra (Pasquali et al., 2016a) (Figura 2). 


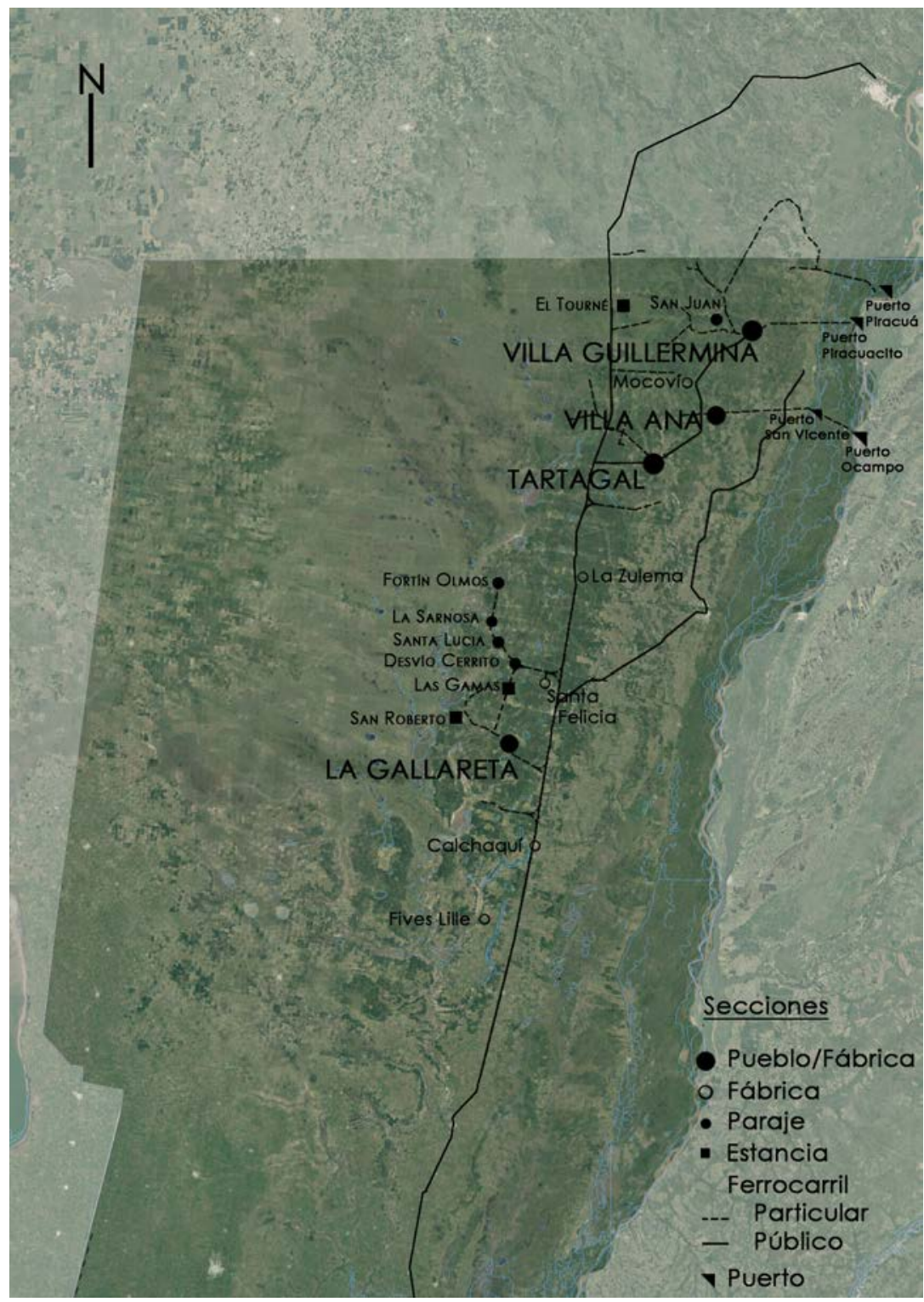

Figura 2. Paisaje industrial

\section{La investigación}

La investigación se realizó en el marco de la Arqueología Histórica (Orser, 1996). El relevamiento de antecedentes de investigación, junto con la búsqueda y análisis de documentación primaria, permitieron profundizar el proceso de configuración histórica del territorio norte de la provincia de Santa Fe (Ogilvie, 1910; Hicks, 1956; Gori, 1965; Bunstorf, 1982; Viñuales, 1983; Gallo, 1984; Regalsky, 1986; Dalla Corte, 2012; Bitlloch y Sormani, 2012; Quarín y Ramírez, 2005; Brac, 2006; Zarrilli, 2008; Jasinsky, 2013; entre otros).

Los trabajos de campo arqueológico tuvieron como objetivo la localización de restos de estructuras, inmuebles y artefactos para, de esta manera, establecer las relaciones espaciales y temporales entre redes 
ferroviarias públicas y particulares, poblados ferroviarios, fábricas/pueblos, parajes y puertos que conformaron el paisaje industrial desde fines del siglo XIX hasta mediados de la década de 1960 (Palmer y Neaverson, 1998).

Los trabajos de campo en los "pueblos tanineros" tuvieron como objetivo identificar las Company towns originales (Garner, 1992; Borges, 2012) en las plantas urbanas actuales, es decir, lo que se conoce como el "pueblo forestal” diferenciado del llamado "pueblo nuevo", sector urbanizado posteriormente, adyacente y periférico al primero. Se realizaron relevamientos de unidades domésticas y equipamientos colectivos. En relación a las fábricas se determinaron estructuras originales y ampliaciones, diferenciándolas de otras que fueron producto de reutilizaciones industriales posteriores. Además se efectuaron reconstrucciones digitales en base a las estructuras disponibles, documentos históricos y fotografías aéreas (Pasquali et al., 2016a). Finalmente, se realizaron prospecciones amplias en terraplenes del F.C.S.F. y en ramales particulares de las distintas compañías y se identificaron estaciones, poblados ferroviarios, parajes y emplazamientos portuarios.

La implementación de talleres, jornadas y entrevistas tuvieron como objetivo indagar en las poblaciones locales sobre el imaginario en relación al pasado forestal: memorias, construcción identitaria y materialización de la memoria en bienes patrimoniales (Norá, 1984; Jelin, 2001). La investigación documentó el valor histórico, arquitectónico y simbólico del patrimonio cultural del norte santafesino como evidencia del pasado, identidad en el presente y potencialidad a futuro en tanto recurso para el desarrollo de las localidades (Pasquali et al., 2016b; Pasquali et al., 2017).

\section{Patrimonio industrial: ¿Qué inventariamos?}

El patrimonio industrial se compone de los restos de la cultura material que poseen valor histórico, tecnológico, social y arquitectónico. Los restos consisten en edificios, maquinarias, fábricas, medios de transporte y toda su infraestructura así también como los sitios donde se desarrollaron actividades sociales relacionadas con la industria, tales como la vivienda, el culto religioso y la educación (Carta de Nizhny Tagil, 2003). Además de los bienes materiales muebles e inmuebles el patrimonio industrial incluye las dimensiones intangibles tales como el conocimiento técnico, la organización del trabajo y los trabajadores (Principios de Dublin, 2011).

Los testimonios del pasado industrial constituyen un legado para conocer y comprender el pasado reciente y el presente del norte santafesino. El paisaje industrial marcó el territorio de forma definitiva y a pesar de ser continuamente despojado forma parte de la identidad de las comunidades del norte provincial.

El inventario propuesto incluye inmuebles de las distintas secciones relacionadas al funcionamiento de la industria para la elaboración de extracto de tanino, es decir, parajes y obrajes para la obtención de la materia prima; fábricas para procesar los rollizos y transformarlos en extracto; pueblos para la residencia de administradores, empleados y obreros; estancias ganaderas para el suministro de carne; redes ferroviarias para conectar y transportar pasajeros y productos $\mathrm{y}$, finalmente, puertos destinados para la exportación del tanino (Pasquali et al., 2018).

\section{Las fichas de inventario}

Los encabezados de las fichas de inventario de las distintas secciones comparten los siguientes campos: Localidad, Departamento, Tipo de Inmueble, Tipo de construcción ${ }^{5}$, Año de Construcción, Uso 
Actual, Estado de Conservación 6 , Plano, Fotografía Histórica y Fotografía Actual. Incluyen además un campo para la ubicación de los inmuebles en el plano de las localidades o en áreas rurales (Figura 3).

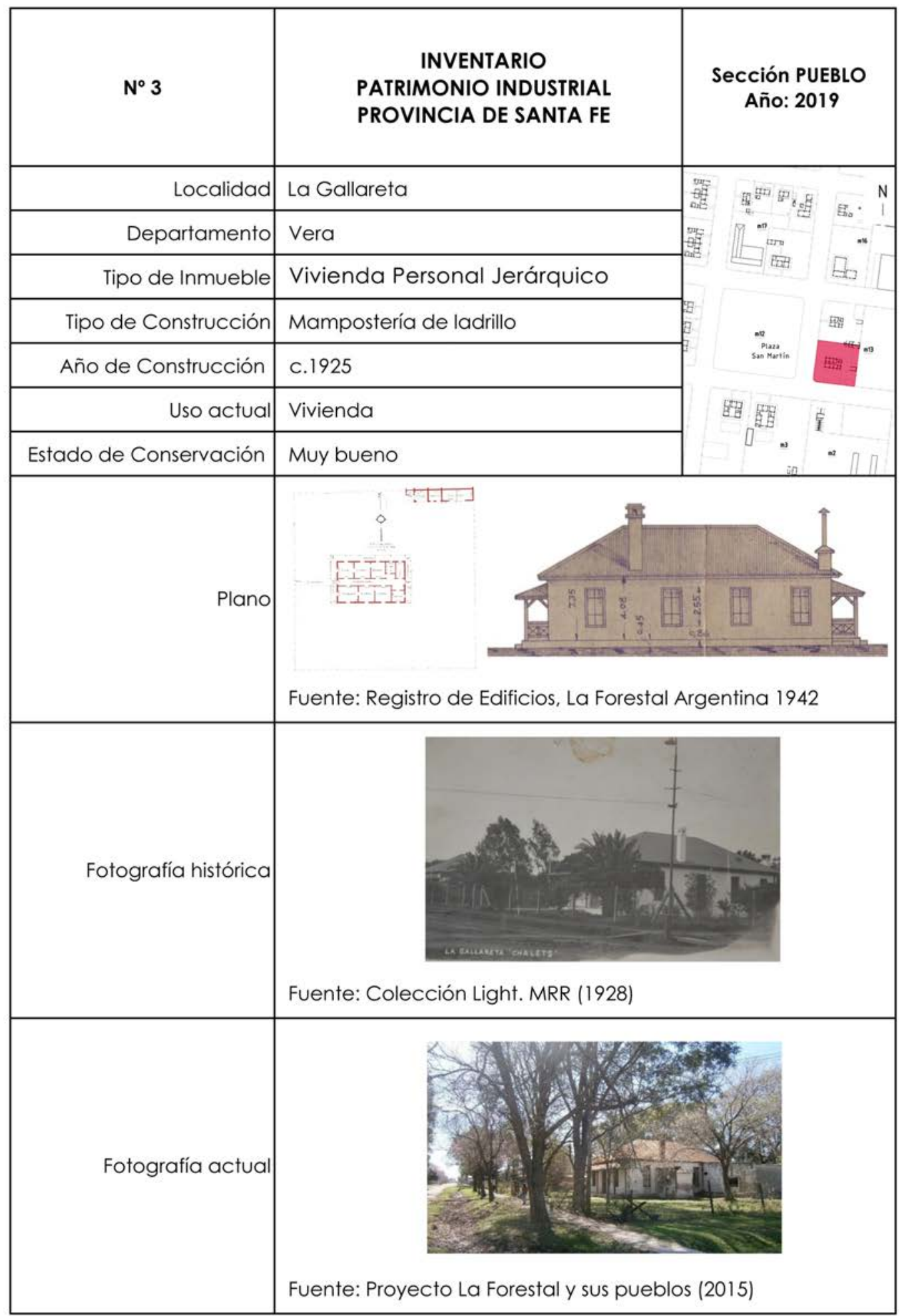

Figura 3. Modelo de ficha de inventario Sección Pueblo 


\section{Sección Fábrica}

La fábrica era el lugar donde se procesaba la materia prima, es decir, los rollizos de Quebracho colorado, para transformarlos en extracto de tanino. La energía para efectuar el proceso estaba dada por las calderas que se alimentaban con residuos de madera. El proceso necesitaba gran cantidad de agua dulce, por lo cual, las compañías construyeron diques o utilizaron lagunas cercanas. Las fichas de inventario incluyen los inmuebles correspondientes al funcionamiento de la industria: Sector Balanza, Playa, Aserrinera, Caldera, Chimenea, Sala de Máquinas, Difusión, Extractería, Depósito, Taller, Usina y Dique. ${ }^{7}$

\section{Sección Pueblo}

La instalación de la fábrica implicaba la construcción de un pueblo, es decir, una urbanización contigua a la fábrica, con viviendas y equipamientos de primera necesidad. Los "pueblos tanineros" eran propiedad de las compañías y en ellos residían administradores, empleados y obreros. La expansión de The Forestal, a partir de 1913, generó la ampliación de las plantas urbanas, la construcción de nuevas viviendas y equipamientos colectivos vinculados a la recreación, deporte y culto religioso. A partir de 1924, la compañía solicitó al Gobierno provincial la aprobación del trazado existente de los pueblos y su ampliación para la posterior venta de tierras. Estas acciones dieron origen a los llamados "pueblos nuevos” donde se instalaron tiendas, bares y almacenes particulares.

Las fichas de inventario de los "pueblos tanineros" incluyen dos tipos de inmuebles: Domésticos: Tipos de viviendas (Personal jerárquico, Empleados y Obreros) y Colectivos: Gestión y Gobierno (Administración/Gerencia, Comisaría, Juzgado de Paz, Correo); Educativo (Escuela); Religioso (Iglesia); Abastecimiento (Almacén, Panadería, Carnicería, Lechería); Alojamiento (Hotel, Casa de Visitas, Solterías); Sanitario (Hospital, Farmacia, Cementerio) y Recreativo (Plaza, Club Social, Club Obrero, Cuartel Boy Scouts, Campo de golf y Cancha de tenis).

\section{Sección Paraje}

Los parajes se situaban sobre las redes ferroviarias y se vinculaban a los obrajes, generando pequeños núcleos urbanos con servicios básicos para sus pobladores. Las fichas incluyen inmuebles Domésticos y Colectivos.

\section{Sección Estancia}

Las estancias se dedicaban a la cría de ganado para abastecer el consumo de carne en los "pueblos tanineros", parajes y obrajes dependientes. Las fichas incluyen tanto los inmuebles domésticos como los distintos sectores vinculados a las actividades productivas.

\section{Sección Ferrocarril}

La instalación de la red del F.C.S.F. (1892) fue crucial para el inicio de la industria taninera. Las redes particulares conectaban fábricas, pueblos, obrajes, estancias y puertos desde donde se exportaba el extracto de tanino. Dichos ramales eran móviles al igual que los obrajes y su ubicación dependía de la disponibilidad del Quebracho colorado aunque siempre ligados al ramal público del F.C.S.F. Esta red 
permitía la exportación del tanino al mercado mundial desde los puertos particulares, Colastiné y Rosario. Las fichas incluyen los distintos tipos de inmuebles relacionados a la actividad ferroviaria: poblados ferroviarios, estaciones, puentes, viviendas del personal y galpones.

\section{Sección Puerto}

Si bien el objetivo de las fichas de inventario es registrar bienes inmuebles, en la sección Puerto, no es posible ya que no se conservan estructuras portuarias. La finalidad de las fichas es presentar el lugar del emplazamiento y fotografías históricas para completar el inventario del paisaje industrial pasado.

\section{Consideraciones finales}

El patrimonio industrial debe entenderse como parte del patrimonio cultural en general y su protección legal debe tener en cuenta su naturaleza especial de acuerdo a la Carta de Nizhny Tagil (2003). Las fichas de inventario presentadas tienen como objetivo preservar digitalmente la información de los inmuebles que conformaron el paisaje industrial del norte santafesino para su difusión y para desarrollar estrategias y políticas de gestión en torno al patrimonio. ${ }^{8}$ Muchos inmuebles fueron y son intervenidos y, en algunos casos, demolidos a pesar de la existencia de legislaciones locales en relación a la protección de bienes patrimoniales. $^{9}$

La implementación de políticas públicas que impulsen el turismo regional permitiría el desarrollo de las localidades a través de una ruta regional y temática que enlace los distintos emplazamientos que constituyeron el paisaje industrial pasado. La propuesta no solo contribuiría a preservar dicho patrimonio, sino también, potenciaría su valor como instrumento didáctico para el conocimiento de las localidades y como medio para reflexionar sobre el pasado industrial y la preservación del medio ambiente (Casanelles Rahola, 2004).

Finalmente, la valorización del patrimonio industrial debería incluir la reutilización de edificios, como los galpones de las fábricas o las estaciones de ferrocarril para la planificar y diseñar centros de interpretación que puedan exhibir distintos tipos de bienes muebles: artefactos, herramientas y fotografías, y en segundo lugar, la preservación de elementos individuales como las chimeneas en tanto símbolos de la identidad de acuerdo a las memorias colectivas de los “pueblos tanineros”.

\section{Notas}

${ }^{1}$ Este artículo se desarrolla en el marco del proyecto de investigación La Forestal y sus pueblos radicado en el Centro de Estudios de Arqueología Histórica. F.H.yA. U.N.R.

${ }^{2}$ El francés E. Dubosc había instalado, en 1862 en Le Havre (Francia), una planta para la producción de extracto de madera para curtir. A partir de la Exposición Universal de Viena (1873) patenta por 15 años la producción de extracto a partir de la madera de Quebracho colorado. Las importaciones de dicha madera, inexistentes en Europa antes de 1873, ascendieron a 85.000 toneladas en 1891, de las cuales la mayor parte era procesada por la firma Dubosc (Huret, 1911). A partir de la Exposición Universal de Paris, en 1889, las fábricas de Alemania y Estados Unidos reemplazaran el Roble y el Hemlock respectivamente por el Quebracho colorado. Comenzaba así la exportación a gran escala de los concesionaros de bosques en el Chaco Austral a las fábricas europeas y norteamericanas.

${ }^{3}$ En 1883, Murrieta le vende a la casa bancaria Kohn, Reinach \& Co (Paris) la mitad de las tierras ad- 
Teoría y Práctica de la Arqueología Histórica Latinoamericana | Año VIII. Vol. 9 | 2019

ISSN en línea: 2591-2801 | ISSN versión impresa: 2250-866X

quiridas en la provincia de Santa Fe y juntos forman la Santa Fe Land Company. Los negocios de la compañía se dividieron en 3 secciones: venta y alquiler de tierras, ganadería y el comercio de madera (Ogilvie, 1910).

${ }^{4}$ La elaboración de extracto de tanino en Argentina a escala industrial comenzó en la provincia de Corrientes, entre 1890 y 1894, en la localidad de Pehuajó. El alemán Schmidt asociado con la firma Herwig de Hamburgo levantaron la primera fábrica (La Forestal al servicio de la grandeza argentina, 1967). Otras fábricas no llegaron a constituirse como pueblos: Fives Lille (Departamento San Justo) perteneciente a la compañía Quebracho S.A, La Zulema propiedad de B. Pinasco (Departamento General Obligado) y Mocoví perteneciente a la Mocoví Tannin Company (Departamento General Obligado), entre otras.

${ }^{5}$ Es importante señalar las características constructivas de los inmuebles inventariados. Si bien están asociadas al destino de cada inmueble, por ejemplo, la mampostería de ladrillos para las viviendas jerárquicas, también están asociadas a la época en que se construyeron, si fueron construidas en serie o solo fueron modelos unitarios.

${ }^{6}$ Se evaluará de acuerdo a las condiciones del inmueble: Estado óptimo (Muy bueno), Necesita arreglos (Bueno) y Pérdida de material (Malo).

${ }^{7}$ Fábricas: La Gallareta, Villa Guillermina, Tartagal, Villa Ana y Santa Felicia. Esta última fue originalmente Departamento de Maderas de Santa Fe Land Company. Entre 1914 y 1923 funcionó en Santa Felicia una fábrica de elaboración de extracto de tanino perteneciente The Forestal. Con el cierre de la fábrica, en 1923, el pequeño pueblo quedó deshabitado. Actualmente funcionan en el paraje el Centro Operativo Forestal, la Unidad Penal 10 y el Cuartel Central de la Guardia Rural “Los Pumas". De la fábrica solo quedan algunas estructuras y cimientos al igual que enTartagal y Mocoví.

${ }^{8}$ Si bien la provincia de Santa Fe carece de una Ley de Patrimonio Arquitectónico, la Ley Orgánica de Ministerios del Poder Ejecutivo, N¹3509/2015, contempla la administración, registro, conservación, defensa y difusión del patrimonio histórico, artístico y cultural de la provincia y otorga a la Secretaría de Producciones, Industrias y Espacios Culturales el deber de implementar políticas públicas con el objeto de preservar, resguardar, registrar, valorizar y difundir el patrimonio histórico y cultural tangible e intangible (Decreto No 172/2015).

${ }^{9}$ La Comisión Comunal de La Gallareta sanciona, en 1995, una Ordenanza $(\mathrm{S} / \mathrm{N})$ en relación a la conservación y puesta en valor de los edificios y documentos de su pasado forestal y el Decreto N²694/2008 aprobando la Ley N¹2927/2008 para la expropiación de 9 inmuebles históricos. En relación a Villa Ana, el Decreto No 2629/1999, declara Monumento Histórico Provincial al predio fabril.

\section{Referencias bibliográficas}

BITLLOCH R. y H. SORMANI (2012). Formación de un sistema productivo: los enclaves forestales de la región chaqueño-misionera (Siglos XIX-XX). Revista de Indias. LXXII (255), 551-580

BORGES, M. J. (2012). Company towns: concepts, historiography, and approaches. En Company Towns: Labor, Space, and Power Relations across Time and Continents. Editado por Borges M. y S. Torres. (pp. 1-40). Washington. Estados Unidos. Palgrave Macmillan

BRAC, M. (2006). La industria del quebracho colorado. Trabajo y vida cotidiana en los Pueblos de La Forestal. Tesis de Licenciatura. Buenos Aires. Argentina: Universidad Nacional de Buenos Aires.

BUNSTORF, J. (1982). El papel de la industria taninera y de la economía agropecuaria en la ocupación del espacio chaqueño. Instituto de Historia. Resistencia. Argentina: Facultad de Humanidades. 
Teoría y Práctica de la Arqueología Histórica Latinoamericana | Año VIII. Vol. 9 | 2019

ISSN en línea: 2591-2801 | ISSN versión impresa: 2250-866X

Universidad Nacional del Nordeste.

CARRANZA, A. (1884). Expedición al Chaco Austral. Buenos Aires. Argentina: Imprenta Europea y Taller.

CASANELLES RAHOLA, E. (2004). Recuperación y uso del patrimonio industrial. Cuadernos del CLAEH 88 (2), 57-64. Montevideo: Uruguay.

DAlla CORTE, G. (2012). Empresas y tierras de Carlos Casado en el Chaco Paraguayo. Historias, negocios y guerras (1860-1940). Asunción. Paraguay: Intercontinental Editora.

FRANCHINI, T. Y J. ROZE (1976). Pueblos Tanineros del Noroeste Argentino Fontana (4). Resistencia: Argentina. Documentos de Arquitectura Nacional y Americana.

GALLO E. (1984). La Pampa Gringa. La colonización agrícola en Santa Fe (1870-1895). Buenos Aires. Argentina: Editorial Sudamericana.

GARNER, J. (1992). The company town: architecture and society in the Early Industrial Age. New York. Estados Unidos: Editorial Oxford Univerty Press

GORI, G. 2006 (1965). La Forestal. La tragedia del quebracho colorado. Santa Fe. Argentina: Mauro Yardín Ediciones.

HICKS, A. (1956). The story of the Forestal. The Forestal Land, London. England: Timber and Railways Company, Limited.

HURET, J. (1911). La Argentina. De Buenos Aires al Gran Chaco. Paris. Francia: Sociedad de Ediciones Louis Michaud.

JASINSKI, A. (2013). Revuelta obrera y masacre en La Forestal. Sindicalización y violencia empresaria en tiempos de Yrigoyen. Buenos Aires. Argentina: Editorial Biblos.

JELIN, E. (2001). ¿De qué hablamos cuando hablamos de memorias?. En: Los trabajos de la memoria (2). España.: Siglo Veintiuno Editores.

LA FORESTAL al servicio de la grandeza argentina (1966). La Forestal Argentina. . Buenos Aires. Argentina: Sociedad Anónima Industrial, Comercial y Agropecuaria

MAGGI, R. (2014). Ferrocarriles y puertos en el norte santafesino. Siglo XIX. En 4 Simposio Nacional e Internacional. Teoría y práctica de la Arqueología Histórica Latinoamericana. Organizado por el Centro de Estudios de Arqueología Histórica. Facultad de Humanidades y Artes. Universidad Nacional de Rosario. Rosario: Argentina.

NORA, P. (1984). Les lieux de mémoire. Paris: Francia: Gallimard.

OGILVIE, C. (1910). Argentina from a British point of view. London. England. Wertheimer, Lea \& Co.

ORSER, C. (1996). A Historical Archaeology of the Modern World. New York. U.S.A: Plenum Press.

PALMER, M. y NEAVERSON, P. (1998). Industrial Archaeology: Principles and Practice. London and New York: Routledge 
PASQUALI, C.; L. FERRÉ, L.; MELETTA, E. y MILICIC, P. (2016 a). ¿Una Company town en el norte santafesino? La Gallareta, Pueblo Forestal (Provincia de Santa Fe). Recuperado en: http:// congresoscnaa.org/web/pagina_submenu.php?opcion=109\&id_menus=2\&id_submenu=109. P. $1035-1041$

PASQUALI, C.; FERRÉ, L., MELETTA, H. y MILICIC, P. (2016 b). Historia y memorias en La Gallareta (1906-2016) (Provincia de Santa Fe). Recuperado en: http://congresoscnaa.org/web/pagina_submenu.php?opcion=109\&id_menus=2\&id_submenu=109. P. 1028-1034

PASQUALI C.; FERRÉ, L. y MILICIC, P. (2017). El patrimonio industrial de los "Pueblos Forestales" (Provincia de Santa Fe). Encuentro de Comités Latinoamericanos de ICOMOS del Siglo XX. Patrimonio Industrial. Buenos Aires. En prensa.

PASQUALI C.; L. FERRÉ, L. y MILICIC, P. (2018). Re- valorizando el patrimonio industrial. Pueblos tanineros de la provincia de Santa Fe (Argentina). En XVII Congreso de TICCIH (Comité Internacional para la Conservación del Patrimonio Industrial). Libro de Resúmenes. P.409-412. Santiago de Chile. ER Impresores

QUARÍN D. y RAMÍREZ., C. (2005). La Gallareta. Una mirada histórica en el año de su Centenario. Comuna de La Gallareta. Santa Fe. Argentina: Imprenta Oficial de la Provincia de Santa Fe.

RAGONESE, A. y CASTIGLIONI, J. (1970). La vegetación del Parque chaqueño. Boletín de la Sociedad Argentina de Botánica 11 (133-166).

REGALSKY, A. (1986). Las inversiones extranjeras en la Argentina 1860-1914. Buenos Aires: Centro Editor de América Latina.

SEELSTRANG, A. 1878 [1977]. Informe de la Comisión Exploradora del Chaco. Buenos Aires. Argentina: Editorial Universitaria de Buenos Aires

VIÑUALES, G. (1983). Los Poblados de la explotación forestal. En Construcción de la Ciudad. La colonización del territorio argentino 1875-1925. 19 (32-35). Barcelona. España.

ZARRILLI, A. (2008). El oro rojo. La Industria del tanino en Argentina (1890-1950). Silva Lusitana 16 (2), 239-259. Lisboa. Portugal. E.F.N.

CARTA DE NIZHNY TAGIL SOBRE EL PATRIMONIO INDUSTRIAL (2003). Recuperado en: https:// www.icomos.org/

PRINCIPIOS PARA LA CONSERVACIÓN DE INDUSTRIALES PATRIMONIO, ESTRUCTURAS, ZONAS Y PAISAJES. Recuperado en: http://www.ticcih.org/

Recibido: 30 de abril de 2019

Aceptado: 14 de agosto de 2019 